Musées, Patrimoine et Culture scientifiques et techniques

$167 \mid 2016$

septembre-octobre 2016

\title{
Le Projet scientifique et culturel aujourd'hui
}

\section{Marie-Hélène Joly}

URL : http://journals.openedition.org/ocim/1703

DOI : $10.4000 /$ ocim. 1703

ISSN : 2108-646X

\section{Éditeur}

OCIM

Édition imprimée

Date de publication : 1 septembre 2016

Pagination : 40-42

ISSN : 0994-1908

Référence électronique

Marie-Hélène Joly, "Le Projet scientifique et culturel aujourd'hui », La Lettre de I'OCIM [En ligne],

167 | 2016, mis en ligne le 01 septembre 2017, consulté le 02 mai 2019. URL : http://

journals.openedition.org/ocim/1703; DOI : 10.4000/ocim.1703

Ce document a été généré automatiquement le 2 mai 2019.

Tous droits réservés 


\title{
Le Projet scientifique et culturel aujourd'hui
}

\author{
Marie-Hélène Joly
}

L'article (disponible sur www.ocim.fr, rubrique La Lettre de l'OCIM) rappelait les principaux objectifs du PSC et insistait sur la nécessité de son évolution.

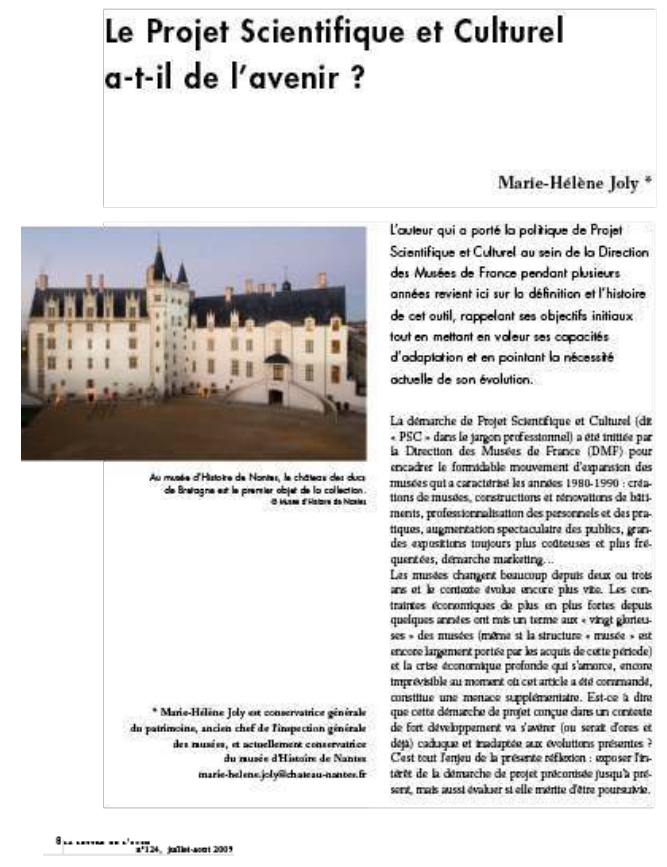

1 Depuis 2009, date du précédent article dans La Lettre de l'OCIM, le projet scientifique et culturel (PSC) en lui-même n'a guère changé. Mais l'environnement culturel et économique a changé, et il convient de se demander en quoi cela peut affecter cette démarche aujourd'hui bien établie'1. 


\section{Un outil de la politique de l'État avant tout}

2 Le PSC reste avant tout un outil de la politique muséale de l'État. "Inventé" voici près de 25 ans par la Direction des Musées de France et progressivement imposé comme clé pour les subventions de l'État aux projets architecturaux, il n'a trouvé son fondement règlementaire qu'avec les décrets d'application de la loi de 2002 sur les Musées de France (aujourd'hui codifiée au livre IV du Code du Patrimoine). Ces décrets ont introduit l'obligation de rédiger un "document d'orientation" pour toute demande d'appellation (art. R. 442-1), sorte de PSC en réduction ${ }^{2}$ et un PSC pour toute demande de subvention à l'investissement (art. D. 442-15)3. Depuis 1992, avec ou sans fondement règlementaire, la Direction des Musées de France (DMF) puis le Service des Musées de France (SMF) ainsi que les conseillers pour les musées des Directions régionales des Affaires culturelles (DRAC) se sont investis dans la formation initiale et permanente, la diffusion de méthodologies et l'accompagnement à l'élaboration. Leur engagement a permis de faire prendre la greffe durablement dans le milieu professionnel.

3 La loi Liberté de la création, architecture et patrimoine (LCAP), publiée au Journal officiel le 8 juillet dernier, introduit une nouveauté législative en imposant à tous les musées de France la production d'un PSC, au-delà de la demande d'appellation et de la subvention d'investissement, jugés comme des champs trop restrictifs. Cette obligation a été introduite dans l'article sur les missions permanentes des musées de France (actuel article L. 441-2), auxquelles s'ajoute désormais la production d'un PSC comportant un volet éducatif. Cette obligation, qui va de soi, a été introduite à la demande des parlementaires.

4 Le PSC devient donc exigible de tous les musées de France, ce qui renforce le contrôle de l'État, toutes les missions des musées de France étant susceptibles de contrôle. Il paraît très pertinent d'avoir choisi de renforcer le contrôle sur le PSC, document de synthèse qui couvre toutes les activités du musée. S'il faut y voir clairement un outil de contrôle, il ne s'agit assurément pas d'une vérification de la conformité à des normes ni d'un contrôle administratif tatillon. À dire vrai, pour avoir vu fonctionner le système au fil des années, il s'agit plutôt d'un accord sur les objectifs de l'établissement, reposant sur la reconnaissance par l'État de la validité et de la justesse de l'analyse et des propositions. La question des critères de validation par l'État a parfois été posée et n'a pas été abordée de front par le SMF, mais s'agissant de la validation d'un projet sous forme textuelle, elle peut difficilement être mise en formule. Il s'agit essentiellement d'évaluer la validité et la justesse de la proposition dans son environnement, ainsi que la cohérence avec le Code $d u$ Patrimoine et avec les grandes politiques de l'État (par exemple ces dernières années l'éducation artistique et culturelle, l'accessibilité, l'action en direction des publics du champ social...). Le PSC apparaît donc plutôt comme un "référentiel" plutôt confortable pour les deux parties (État/musée et son autorité de tutelle), dans le cadre d'un partenariat qui facilitera l'obtention de subventions. Les moyens d'intervention de l'État ayant beaucoup baissé, tant dans le domaine de l'investissement que du soutien aux acquisitions et aux activités de conservation et en direction des publics, l'existence du PSC sera une aide accrue à la décision pour l'État. Par ailleurs, le contrôle n'est pas forcément à envisager de façon négative: même s'il n'a pas abordé précisément la question du PSC, le Livre blanc des musées, publié en 2011 par l'Association générale des 
conservateurs des collections publiques de France, appelait à plus de contrôle de l'Étatarbitre pour contrebalancer certaines dérives.

5 Une autre évolution mineure est envisagée à l'heure actuelle par le SMF : les PSC "initiaux" seront encore validés par l'administration centrale alors que les renouvellements de PSC seront évalués par les DRAC, ce qui ne devrait pas apporter de changement majeur dans la vie des musées.

\section{Que représente le PSC pour les musées eux-mêmes ?}

6 Aujourd'hui, le PSC fait clairement partie du paysage muséal. Tous les responsables, conservateurs, attachés... y ont été formés, maîtrisent la méthode de rédaction ou savent où trouver l'information. De plus, certains établissements ont déjà rédigé leur deuxième PSC, voire le troisième, le renouvellement étant indispensable au bout de quelques années.

7 Par ailleurs, certains responsables ont choisi d'en rédiger un en dehors de toute contrainte règlementaire. Nous ne savons pas encore comment sera accueilli par les responsables de musées et les autorités de tutelle le fait de devoir en rédiger un, même en dehors du cadre de l'appellation, ou d'une subvention à l'investissement, mais la forme sous laquelle il sera demandé jouera certainement beaucoup (voir infra).

Il est difficile de savoir exactement combien de PSC ont été rédigés depuis le début des années 1990 (du fait d'une grande perte d'information au moment de la révision générale des politiques publiques). Sur les 1220 Musées de France, plusieurs centaines se sont astreints à l'exercice. Une étude récente du bureau des réseaux territoriaux du SMF précise que 73 PSC de musées territoriaux ont été instruits entre 2010 et $2015^{\left({ }^{(4)}\right.}$.

9 Presque tous les musées nationaux du ministère de la Culture en ont rédigé un, alors qu'ils ont été longtemps réfractaires à cette obligation, et les musées nationaux du ministère de la Défense ont fait de même.

10 La démarche a été clairement appropriée par la profession, mais il est intéressant de noter que l'initiative ne vient plus seulement de l'état ou de responsables convaincus ou consciencieux, mais qu'elle a été intégrée aussi par nombre d'autorités de tutelle: les directeurs des affaires culturelles des grandes collectivités territoriales connaissent le processus et il n'est pas rare de voir en tête d'un avis de vacance pour un poste de directeur de musée la demande d'élaboration du PSC de l'établissement.

\section{Vers une évolution de la forme du PSC ?}

11 À l'usage, l'utilité du PSC ne paraît pas contestable. Même si désormais les chantiers de musées sont beaucoup moins nombreux, le PSC reste indispensable dans le cas d'une création ou d'une rénovation architecturale importante, pour énoncer ce que l'on veut faire et en déduire les caractéristiques futures de l'établissement et du bâtiment. Il est évidemment indispensable dans le cadre d'une demande d'appellation, puisque c'est le document d'orientation qui permet au Haut conseil des Musées de France d'apprécier les lignes de force du futur établissement et donc la recevabilité et la viabilité de la demande. En dehors de ces deux obligations règlementaires, la démarche ne peut être que fructueuse (réfléchir, proposer, énoncer, consulter, s'inscrire dans les axes des politiques de l'autorité de tutelle, associer les équipes et les partenaires pour élaborer un projet 
véritablement collectif et pertinent), sans oublier son rôle pédagogique notamment auprès de la tutelle, par l'intégration de toutes les missions (même celles qui paraissent basiques à un conservateur). Sa plasticité, adaptée à toutes les formes et situations, n'est plus à prouver, et l'individualité d'un territoire comme la créativité d'une équipe peuvent trouver à s'y exprimer sans mal.

En revanche, il paraît indispensable de promouvoir une rédaction beaucoup plus stratégique énonçant clairement et précisément les axes prioritaires, en se limitant à une forme plus courte. Le SMF a admis récemment cette notion de PSC allégé, mais sans diffuser jusqu'à présent d'instructions subséquentes. Il paraît même nécessaire d'aller plus loin, en suggérant une rédaction d'une dizaine de pages, privilégiant le projet sur le bilan (en faisant crédit aux rédacteurs de la connaissance de leur établissement) et l'énonciation des axes stratégiques sur la forme rédactionnelle. Par ailleurs, il paraît plus opérationnel de limiter le champ chronologique du PSC à 3 à 5 ans maximum (tout en situant l'évolution du musée dans une perspective longue) car, au-delà, la démarche paraît plus aléatoire.

\section{Quelles limites au PSC?}

Dans le meilleur des mondes, le PSC reste un instrument très utile. Mais son principal défaut est qu'il n'est pas un document contractuel. Au mieux, nous pouvons considérer qu'il constitue une forme de contrat moral avec l'État qui l'a validé et peut sur cette base attribuer des subventions, même modestes. Mais la relation à l'autorité de tutelle reste fondamentale, et il n'existe pas de contractualisation avec cette dernière (sauf dans le cadre des établissements publics, très rares à l'échelon local).

Le PSC souffre d'un problème de concordance entre le temps long du patrimoine et le temps court du politique. Cela a toujours été le cas, mais la conjoncture économique défavorable peut aujourd'hui entraîner de fortes réductions budgétaires, et l'élection d'une nouvelle équipe municipale peut mettre en cause la place et le rôle du musée comme outil culturel et social dans la cité, même si l'autorité de tutelle a validé un PSC. $\mathrm{Au}$ mieux peut-il jouer le rôle d'amortisseur, à condition que le choc ne soit pas trop fort. Il est inquiétant de voir que le nombre de musées fragilisés va croissant depuis quelques années (réductions drastiques de budgets, non renouvellement du personnel scientifique, voire à l'extrême menaces de fermeture). Le cas du musée des manufactures de dentelle de Retournac (Haute-Loire) est à cet égard tristement emblématique : créé ex nihilo à la fin des années 1990 avec une belle collection (textiles, bâtiments industriels et machines), parfaitement intégré à son territoire, aménagé avec l'aide de l'état et de toutes les collectivités territoriales, inauguré en 2007 et développant un programme d'activités ambitieux. Ce musée est réduit à la plus grande pénurie financière et culturelle depuis 2008, du fait d'un changement de municipalité doublé de difficultés financières. Le souhait du Maire est de fermer l'établissement, ce que le Code du Patrimoine ne permet pas, à moins de transférer les collections à un autre Musée de France (mais sans repreneur identifié dans ce cas). Et ce constat ne s'applique pas seulement aux musées petits ou moyens comme le montre l'exemple actuel du musée des Tissus de Lyon.

Face à ces situations inquiétantes, l'État ne peut pas grand-chose pour plusieurs raisons : réelles difficultés financières des autorités de tutelle, libre administration des 
collectivités territoriales, absence de capacités d'intervention. Il faut bien reconnaître que le PSC ne pèse d'aucun poids dans ces conditions extrêmes.

Mais, heureusement, toutes les situations ne sont pas extrêmes. Il vaut donc mieux écrire un document stratégique concis, limité dans le temps, qui servira de base au dialogue dans un contexte normal, tout en sachant qu'il faudra peut-être un jour faire évoluer sa stratégie en réduisant la voilure.

\section{NOTES}

1. Ayant quitté depuis 2007 la Direction des Musées de France (devenue le Service des Musées de France), ce que je peux écrire ici n'est qu'une opinion personnelle et non l'expression d'une position institutionnelle.

2. Document précisant les objectifs scientifiques et culturels du futur musée, les conditions et les moyens de leur mise en œuvre en matière de collections, de personnels, de muséographie, d'éducation, de diffusion et de recherche.

3. Dans le cadre des subventions à l'investissement, l'État exige un PSC, un programme de conservation et de présentation des collections et un programme architectural et valide chacun de ces documents. (4) Éléments fournis par le bureau des réseaux territoriaux (BRT) : sur les 88 PSC rédigés par des musées en région et adressés au SMF entre 2010 et 2015, 15 ayant été déclarés caducs par l'autorité de tutelle (abandon de projet, changement de majorité politique), 73 ont été instruits par le BRT. 50 ont été rédigés à l'occasion d'un projet de rénovation ou d'extension; 15 pour une demande d'appellation ; 2 pour une création de musée ; 2 à l'occasion d'une restructuration-fusion de musées ; 1 lors d'un changement de tutelle ; 2 dans le cadre d'une réflexion globale sur l'identité et la vocation du musée ; et enfin une actualisation de PSC ancien. 5 seulement ont reçu un avis défavorable (ce qui n'exclut pas une nouvelle présentation). Par ailleurs, le BRT reçoit aussi des "pré-PSC", documents intermédiaires de travail qui facilitent le dialogue.

\section{RÉSUMÉS}

La rubrique "Quoi de neuf ?" propose un retour sur un article marquant de l'histoire de la revue. À partir d'un corpus d'articles choisis par la rédaction de La Lettre de l'OCIM, les membres du comité des Publications de l'OCIM ont sélectionné plusieurs contributions. Dans cette perspective, il a été demandé à l'auteur ou à un expert du domaine de revisiter la problématique exposée dans l'article à la lueur des changements intervenus, notamment dans les pratiques professionnelles, depuis son écriture et de proposer des éléments prospectifs sur la question.

Marie-Hélène Joly revient sur son article "Le Projet scientifique et culturel a-t-il de l'avenir ?" publié dans le $n^{\circ} 124$ de La Lettre de l'OCIM en juillet-août 2009 et dans lequel elle présentait cet outil, pierre angulaire de la politique muséale de l'État. 
INDEX

Mots-clés : projet scientifique et culturel

\section{AUTEUR}

MARIE-HÉLÈNE JOLY

conservatrice générale du patrimoine, inspection des patrimoines, ministère de la Culture et de la Communication

marie-helene.joly@culture.gouv. 